\title{
Targeting Angiogenesis and Tumor Microenvironment in Metastatic Colorectal Cancer: Role of Aflibercept
}

\author{
Guido Giordano, ${ }^{1}$ Antonio Febbraro, ${ }^{1}$ Michele Venditti, ${ }^{1}$ \\ Serena Campidoglio, ${ }^{1}$ Nunzio Olivieri, ${ }^{2}$ Katia Raieta, ${ }^{3}$ Pietro Parcesepe, ${ }^{4}$ \\ Giusy Carmen Imbriani, ${ }^{5}$ Andrea Remo, ${ }^{6}$ and Massimo Pancione ${ }^{3}$ \\ ${ }^{1}$ Medical Oncology Unit, Ospedale Sacro Cuore di Gesù Fatebenefratelli, 82100 Benevento, Italy \\ ${ }^{2}$ Department of Biology, Federico II University, 80131 Napoli, Italy \\ ${ }^{3}$ Department of Sciences and Technologies, University of Sannio, 82100 Benevento, Italy \\ ${ }^{4}$ Department of Surgical and Diagnostic Pathology, "G.B. Rossi" Hospital, University of Verona, 37134 Verona, Italy \\ ${ }^{5}$ Fifth Division of General Surgery and Special Surgical Techniques, Second University of Studies of Naples, 80138 Naples, Italy \\ ${ }^{6}$ Department of Pathology, "Mater Salutis" Hospital, 37045 Legnago, Italy
}

Correspondence should be addressed to Guido Giordano; giordano.guido81@gmail.com and Massimo Pancione; massimo.pancione@unisannio.it

Received 15 November 2013; Revised 12 April 2014; Accepted 27 April 2014; Published 21 July 2014

Academic Editor: Gianfranco D. Alpini

Copyright (c) 2014 Guido Giordano et al. This is an open access article distributed under the Creative Commons Attribution License, which permits unrestricted use, distribution, and reproduction in any medium, provided the original work is properly cited.

In the last decades, we have progressively observed an improvement in therapeutic options for metastatic colorectal cancer (mCRC) treatment with a progressive prolongation of survival. mCRC prognosis still remains poor with low percentage of 5-year survival. Targeted agents have improved results obtained with standard chemotherapy. Angiogenesis plays a crucial role in colorectal cancer growth, proliferation, and metastasization and it has been investigated as a potential target for mCRC treatment. Accordingly, novel antiangiogenic targeted agents bevacizumab, regorafenib, and aflibercept have been approved for mCRC treatment as the result of several phase III randomized trials. The development of a tumor permissive microenvironment via the aberrant expression by tumor cells of paracrine factors alters the tumor-stroma interactions inducing an expansion of proangiogenic signals. Recently, the VELOUR study showed that addition of aflibercept to FOLFIRI regimen as a second-line therapy for mCRC improved significantly OS, PFS, and RR. This molecule represents a valid second-line therapeutic option and its peculiar ability to interfere with placental growth factor (PlGF)/vascular endothelial growth factor receptor 1 (VEGFR1) axis makes it effective in targeting angiogenesis, inflammatory cells and in overcoming resistances to anti-angiogenic first-line treatment. Here, we discuss about Aflibercept peculiar ability to interfere with tumor microenvironment and angiogenic pathway.

\section{Introduction}

Colorectal cancer (CRC) represents the third most common cancer worldwide with $1,200,000$ newly diagnosed cases each year and the second leading cause of cancer related deaths with 600,000 deaths annually $[1,2]$. Nowadays overall survival in metastatic patients has reached approximately 24 months, mostly thanks to the introduction of targeted agents who have improved efficacy of standard chemotherapy. For more than fifty years, 5-fluorouracil has represented the backbone of all chemotherapy schedules, used both alone and combined. The addition of oxaliplatin and irinotecan to fluorouracil based treatment has increased response rate (RR) and overall survival (OS) [3-5]. Nevertheless, chemotherapy alone reached 18-20 months survival plateau obtained administering alternatively all active cytotoxic agents in the course of treatment strategy [6]. Genetic and molecular studies have led to better understanding of tumor growth, proliferation, differentiation, and metastasization pathways, resulting in the development of molecular targeted agents. Two major pathways are involved in MCRC: the epidermal growth factor receptor (EGFR) cascade and the vascular endothelial growth factor (VEGF) signaling [7]. Randomised 


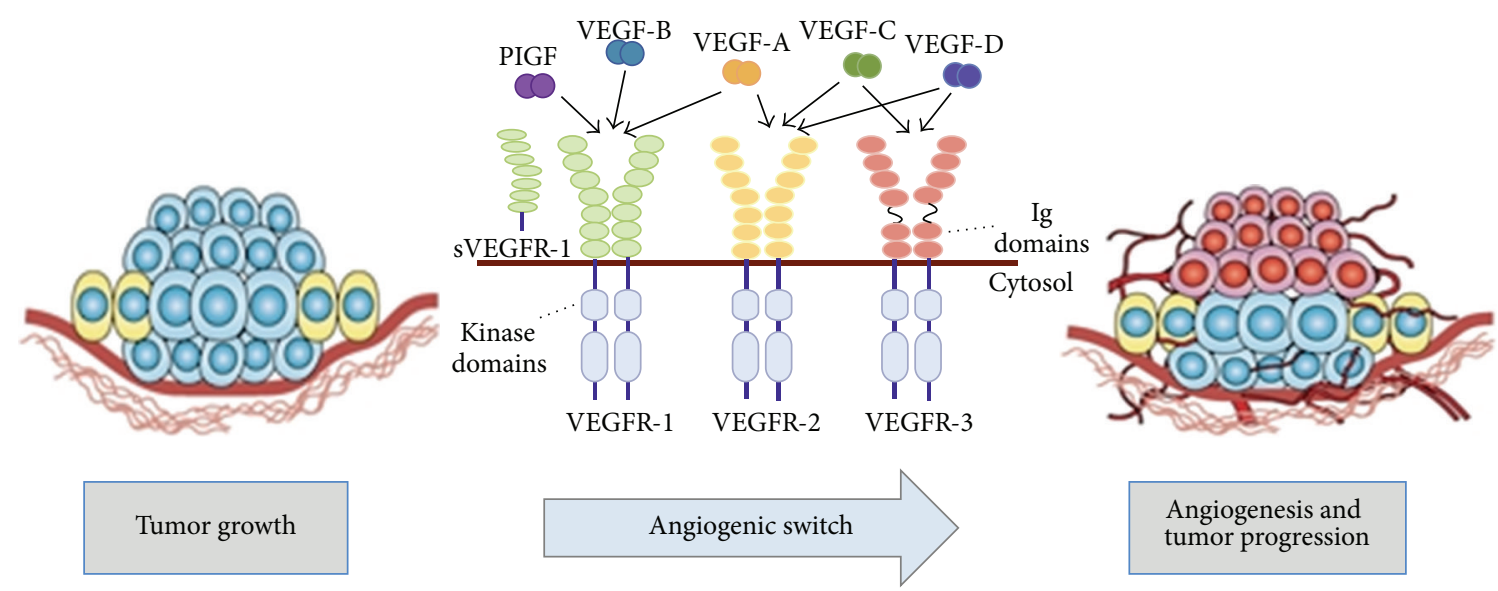

FIGURE 1: Angiogenesis promotes cancer growth and metastasis. Angiogenesis, the process of developing new blood vessels from preexisting vascular networks, is a well-described mechanism leading to the initiation and maintenance of tumours and the promotion of metastasis at secondary sites. The vascular endothelial growth factor (VEGF) family of ligands and receptors mainly includes VEGF-A, VEGF-B, VEGF-C, VEGF-D, placental derived growth factor (PlGF), VEGFR-1, VEGFR-2, and VEGFR-3. The best characterized of the VEGF family members is VEGF-A, whose binding to VEGFR-2 (FLK1) is the predominant mechanism through which tumour cells promote the so-called angiogenic switch.

phase III clinical trials have shown that targeting EGFR with monoclonal antibodies cetuximab and panitumumab has resulted in improved outcomes in mCRC patients with the limitation to Ras wild-type population [8-10]. On the other hand, given the crucial importance of neoangiogenesis in mCRC, anti-VEGF pathway therapies have been intensively investigated [11]. Monoclonal antibody bevacizumab is the first antiangiogenic agent to be approved in mCRC treatment, because of response rate and survival benefit [12]. Recently, new antiangiogenic agents, aflibercept and regorafenib, with mechanisms of action different from bevacizumab, have increased the survival in mCRC patients $[13,14]$.

Growing evidence suggests that the crosstalk between genomic/epigenomic aberrations of malignant cells and the surrounding microenvironment that is composed of immune and stromal cells contributes to the enhancement of tumor growth culminating in metastatic dissemination [15-17]. Over the past decades, it has become increasingly clear that the mobilization of immune cells, such as myeloid derived suppressor cells (MDSCs) and tumor associated macrophages (TAMs) may contribute either to a lack of response or to acquired drug resistance [18]. Recent studies have shown that vascular endothelial growth factor A (VEGF-A) signaling through vascular endothelial growth factor receptor-2 (VEGFR-2) is involved in MDSCs precursors recruitment to metastases and, once within the tumor, MDSCs can further mature into tumor-promoting macrophages. Additional angiogenic factors such as the placental growth factor (PlGF) directly or indirectly stimulate angiogenesis by affecting a wide range of different cell types or by attracting MDSCs and macrophages within the tumor microenvironment (TME). Notably, PlGF activates inflammation and pathological angiogenesis mainly by interacting with alternative pathways via VEGFR-1 signaling. This is a relevant issue given that myeloid cells primarily express VEGFR-1 and not VEGFR2 and PlGF exerts complementary effects, independent of VEGF-A [19, 20]. Myeloid cells have been considered the main mechanism of resistance to anti-VEGF targeted therapies by secreting additional proangiogenic factors [21, 22]. These observations suggest that the cells surrounding the tumor microenvironment expand angiogenic signaling pathways through from VEGF-A; this condition seems to be crucial for tumor outgrowth, regrowth, or metastatic dissemination $[23,24]$.

In this paper, we focus our attention on angiogenesis and its dialogue with TME and antiangiogenic therapies in mCRC, underlining the role of aflibercept and its possible employment in clinical practice.

\section{Tumor Angiogenesis and Its Mediators}

Angiogenesis is defined as the process of blood vessels neoformation through a multistep mechanism that provides nutrients and oxygen to tissues, allowing discharge of waste products [25]. This process works in physiological conditions such as wound healing, embryogenesis, and inflammation, but it is also crucial during pathological conditions like cancer [24]. Angiogenesis is a well-regulated process and, in normal conditions, there is a balanced equilibrium between pro- and antiangiogenic factors as well as between multiple signalling pathways $[26,27]$. Under conditions of malignancy, like during neoplastic processes, there is a disruption of the balance between proangiogenic and antiangiogenic factors better known as "angiogenic switch" driven by the increase of nutrient supply necessary for tumor growth (Figure 1) [28, 29]. VEGFs represent the most important and widely studied proangiogenic factors family and it is composed of 5 growth factors named VEGF-A, VEGF-B, VEGF-C, VEGF-D, and PlGF [30-32]. VEGFs play their role in angiogenesis through binding to three different receptors located on cell membrane: VEGFR-1 (Flt-1), VEGFR-2 (Flk/KDR), and VEGFR-3 (Flt4) (Figure 1). VEGFRs structure consists of an extracellular 
domain that binds specific ligands, a transmembrane domain, and an intracellular region with tyrosine kinase domain [33]. Activities of VEGFRs can be enhanced by neuropilin receptors (NRP-1 and NRP-2) that may act like coreceptors for VEGFs [34]. VEGF-A represents the first member of VEGF family and the most intensively studied. It is also the most important regulator in human physiologic and pathologic angiogenesis and it is related to a poor prognosis in several cancers [35]. VEGF-A may interact with both VEGFR-1 and VEGFR-2, but the major VEGFR-2 intrinsic kinase activity makes it the most important effector of VEGFA downstream signalling [36]. The effects of this interaction are represented by endothelial cell proliferation, survival, migration, invasion, vascular permeability, and vasodilatation. Less is known about the other members of VEGF family. VEGF-B has common structural homology with VEGF-A and its activity, mediated by interaction with VEGFR-1 and NRP-1, could have a role in tumorigenesis and blood vessels survival under stress conditions [37]. VEGF-C and VEGF$\mathrm{D}$ that bind VEGFR-3 are involved in lymphangiogenesis. In particular VEGF-D expression has been associated with tumor metastasization to regional lymph nodes [38]. PlGF is another important growth factor which regulates vessel growth and maturation directly by affecting endothelial and mural cells, as well as indirectly by recruiting proangiogenic cell types (Figure 2). PlGF shares structural homology with VEGF-A and stimulates angiogenesis via interaction with VEGFR-1 downstream signaling [39-42] (Figure 1). In fact, PlGF binding to VEGFR-1 modulates recruitment of bone marrow derived macrophages to the tumor site, where they release proangiogenic factors [43]. There are many evidences that PIGF and VEGFs levels are strongly involved in CRC proliferation and metastasization influencing patients outcome [44-47]. Activation of proangiogenesis factors and/or microvessel density has been related to increased vascularization, advanced disease, and poor prognosis in a variety of tumours $[48,49]$. It has been demonstrated that there is a strong correlation between vascular density in primary colorectal cancer recurrence, metastasis, and mortality [50]. Unlike VEGFA, which binds to both VEGFR-1 and VEGFR-2, PlGF binds to VEGFR-1 but not VEGFR-2 (Figure 1). Several reports have documented that also PIGF and VEGFR-1 are increased in various tumors, which correlate with disease progression and can predict poor prognosis, metastasis, and recurrent disease [51-53]. The concept of angiogenesis as a marker of tumor aggressiveness is enforced by evidences of higher vascular density at the tumor invasion front if compared to other areas within the tumor [53]. Furthermore, an increased expression of VEGF mRNA has been detected in human CRC liver metastasis, and VEGFR-1 and VEGFR2 expression were upregulated in liver metastases compared with contiguous normal liver [54-56].

\section{Relation between Tumor Microenvironment and Angiogenesis}

A general feature of advanced tumors is represented by the altered reciprocal interaction of cancer cells with surrounding nonmalignant cells, soluble inflammatory mediators, and components of the extracellular matrix. Emerging evidence suggests that the interconnected plasticity of tumor cells and stromal cells in response to treatment-induced tumor tissue injury and inflammation represents a common aspect of therapy resistance. One of the mechanisms that tumors utilize to promote metastases and escape antiangiogenic therapies is the activation of inflammatory cells and MDSCs (Figure 2). Consistently, our results (Pancione et al. unpublished data) suggest that VEGFR-1, but not VEGFR-2, is expressed significantly in nonmalignant cells and closely linked with CD68 infiltration "a M2 macrophage marker," suggesting that it may have a greater role than VEGFR-2 in TME (Figure 3(a)). Additional studies support our own results implying that VEGFR-1 expression is higher in liver metastasis tissues than in primary tumours and thus is associated with tumour aggressiveness (Figures 3(a), 3(b), and 3(c)) [57, 58].

According to this, inflammatory mediators and cytokines such as the tumor necrosis factor (TNF)- $\alpha$, transforming growth factor (TGF- $\beta$ ), and PlGF have previously been shown to have a significant effect on the inflammatory neovascularization implicating a prominent role of VEGFR1 in this process $[59,60]$. On the other hand, VEGFR-2 expression is mainly detected in malignant cells of primary CRC. VEGFR-1 but not VEGFR-2 expression is associated with poor survival time when analyzed alone in stages III and IV patients both in our unpublished data (Figure 3(c)) and literature data $[20,57,58]$. Altogether, our data and those reported in literature provide evidence that expression of VEGFR-1 is markedly associated with infiltration of inflammatory cells in the tumors $[19,20,61,62]$. This is supported by the observation that PlGF potentiates the response to VEGF-A by signalling through VEGFR-1, and, in turn, it stimulates the recruitment of bone marrow derived macrophages to the tumour site by enhancing the expression of proangiogenic factors in the TME (Figure 2). PlGF exerts pleiotropic activities in the TME, by interacting specifically with VEGFR-1 on multiple types of vascular and nonvascular cells (Figure 2). Mechanisms of resistance or escape from anti-VEGF therapies (bevacizumab) may be promoted by releasing alternative angiogenic factors such as PlGF in malignant cells and inflammatory cells which might contribute to the induction of an angiogenic rescue program (Figure 2) [63].

\section{Antiangiogenic Drugs in mCRC}

In the last decade, different antiangiogenic strategies have been investigated in preclinical and clinical studies, showing how angiogenesis can be targeted by several approaches $[64,65]$. Nowadays, it is possible to target angiogenesis with monoclonal antibodies which bind to VEGF, preventing its interaction with VEGFRs, with small molecules inhibiting tyrosine kinase (TK) activity of VEGFRs or using newer soluble receptor fragments like VEGF-trap technology (Figure 2) $[66,67]$. Also anti-EGFR monoclonal antibodies cetuximab and panitumumab may have an indirect inhibition effect on angiogenesis [68]. Only three antiangiogenic agents have been approved by regulatory authorities for mCRC treatment: bevacizumab, regorafenib, and aflibercept [69]. 


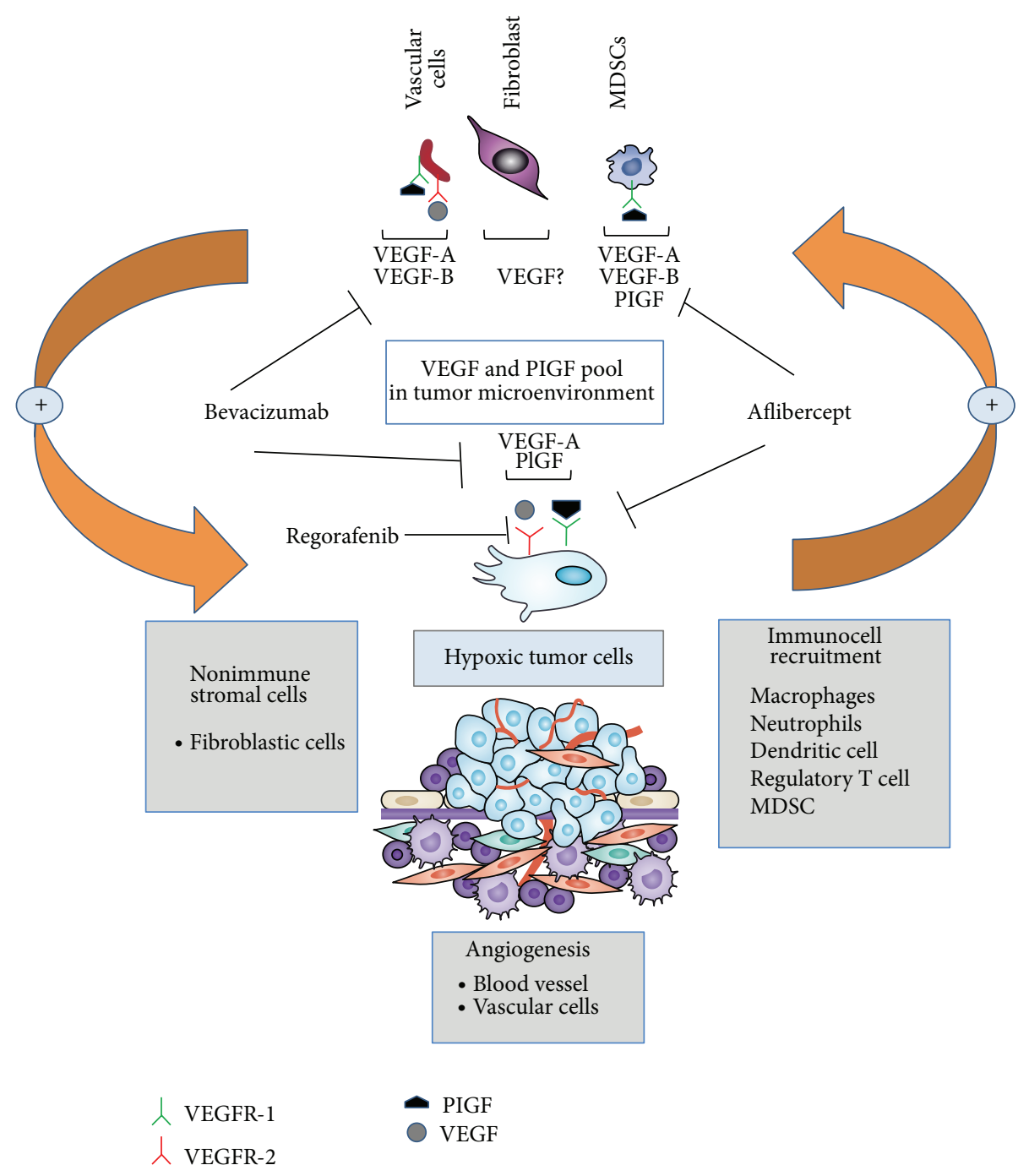

FIGURE 2: New molecules targeting angiogenesis and crosstalks between angiogenesis and tumor microenvironment. Simplified and schematic view of how multiple cells contribute to the VEGF and PlGF pool in the tumor microenvironment. The tumor microenvironment (TME) consists of soluble molecules, immune, nonimmune fibroblastic, vascular, and malignant cells that interact in a paracrine and autocrine fashion to promote cancer growth and metastasis. Hypoxia is the most potent stimulus for inducing the main angiogenic factors, VEGF and PlGF. Myeloid derived suppressor cells (MDSCs) might confer resistance to therapies that target VEGF by secreting additional proangiogenic factors and specifically by expressing VEGFR-1 (also known as FLT1). PlGF signals directly through VEGFR-1 in various cell types, including endothelial cells, angiogenesis-competent myeloid progenitors, macrophages, and tumour cells and thereby promotes tumour growth and the formation of the premetastatic niche. A substantial fraction of tumours is resistant or escapes antiangiogenic inhibitors that target VEGF-A signalling (bevacizumab) through therapy-induced injury, metabolic changes, inflammation, and possibly expansion of MDSCs. Differently from other antiangiogenic drugs, aflibercept targeting PlGF should reduce the source of the compensatory upregulation of angiogenic factors by inhibiting immune cells recruitment and/or polarization and the release of angiogenic factors by tumour and vascular cells. Regorafenib is a multikinase inhibitor against selected tyrosine kinases and signal transduction VEGFR2-3/RAF/MEK/ERK pathway.

4.1. Bevacizumab: Mechanism of Action and Clinical Studies. Bevacizumab, a humanized monoclonal antibody that binds to VEGF-A preventing its interaction with VEGFR-2, was the first antiangiogenic agent approved in mCRC (Figure 2 and Table 1). A randomized phase III trial comparing 5fluorouracil/leucovorin and irinotecan (IFL) regimen alone or in combination with bevacizumab has shown that addition of antiangiogenic molecule improved not only RR (35 versus 45\%) and PFS (6.2 versus 10.6 months) but also OS (15.6 versus 20.3 months) [12]. Also oxaliplatin based regimens have demonstrated survival benefit in combination with bevacizumab in both first- and second-line settings. In the NO16966 phase III trial, addition of bevacizumab to oxaliplatin based schedules with fluorouracil and leucovorin (FOLFOX) or capecitabine (XELOX) prolonged significantly PFS but OS difference was not statistically relevant. In this study, also response rates were similar between bevacizumab containing and not containing arms [70]. In second-line treatment for mCRC, the ECOG E3200 trial showed that addition of bevacizumab to FOLFOX4 regimen increased RR, 

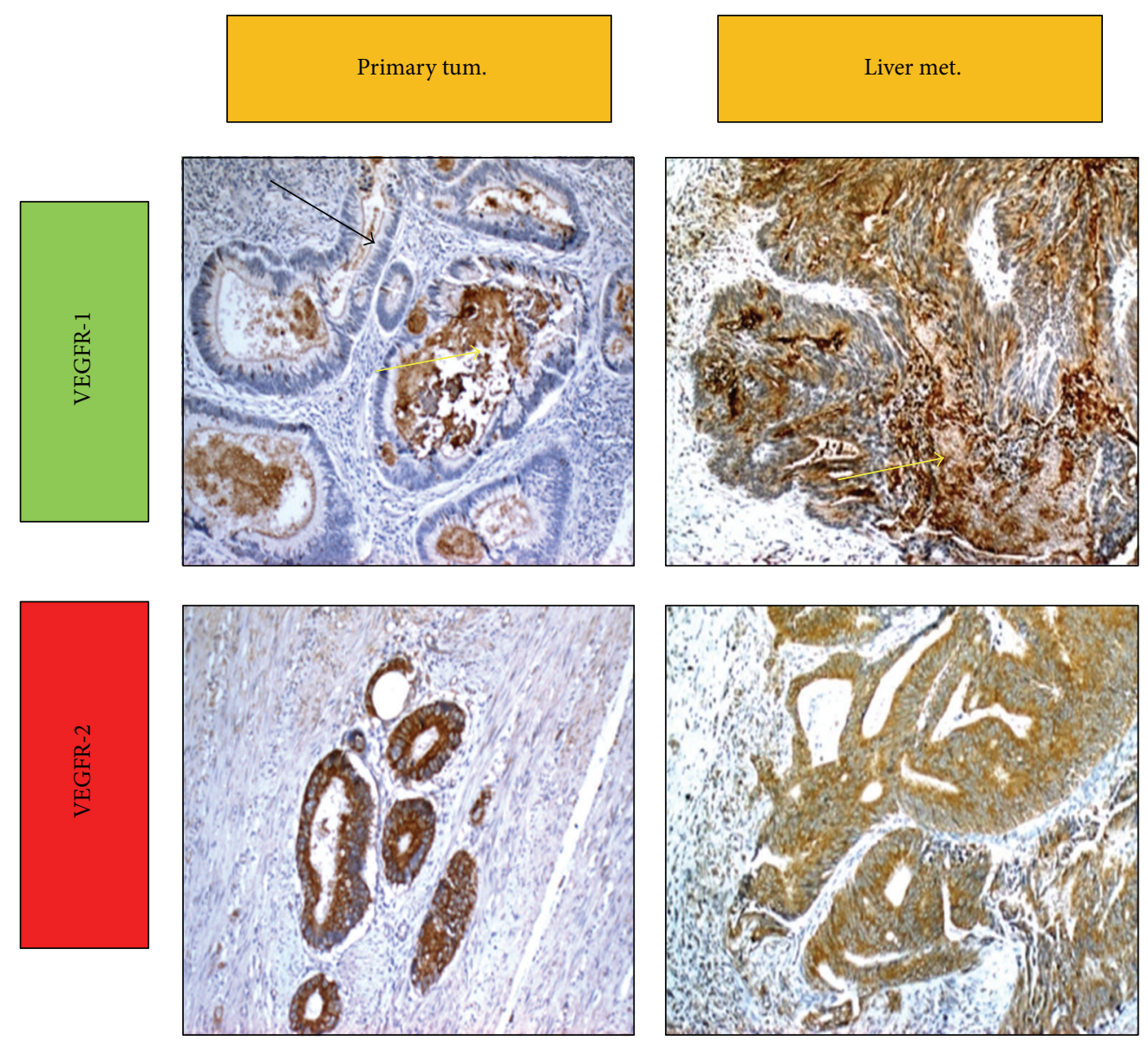

(a)
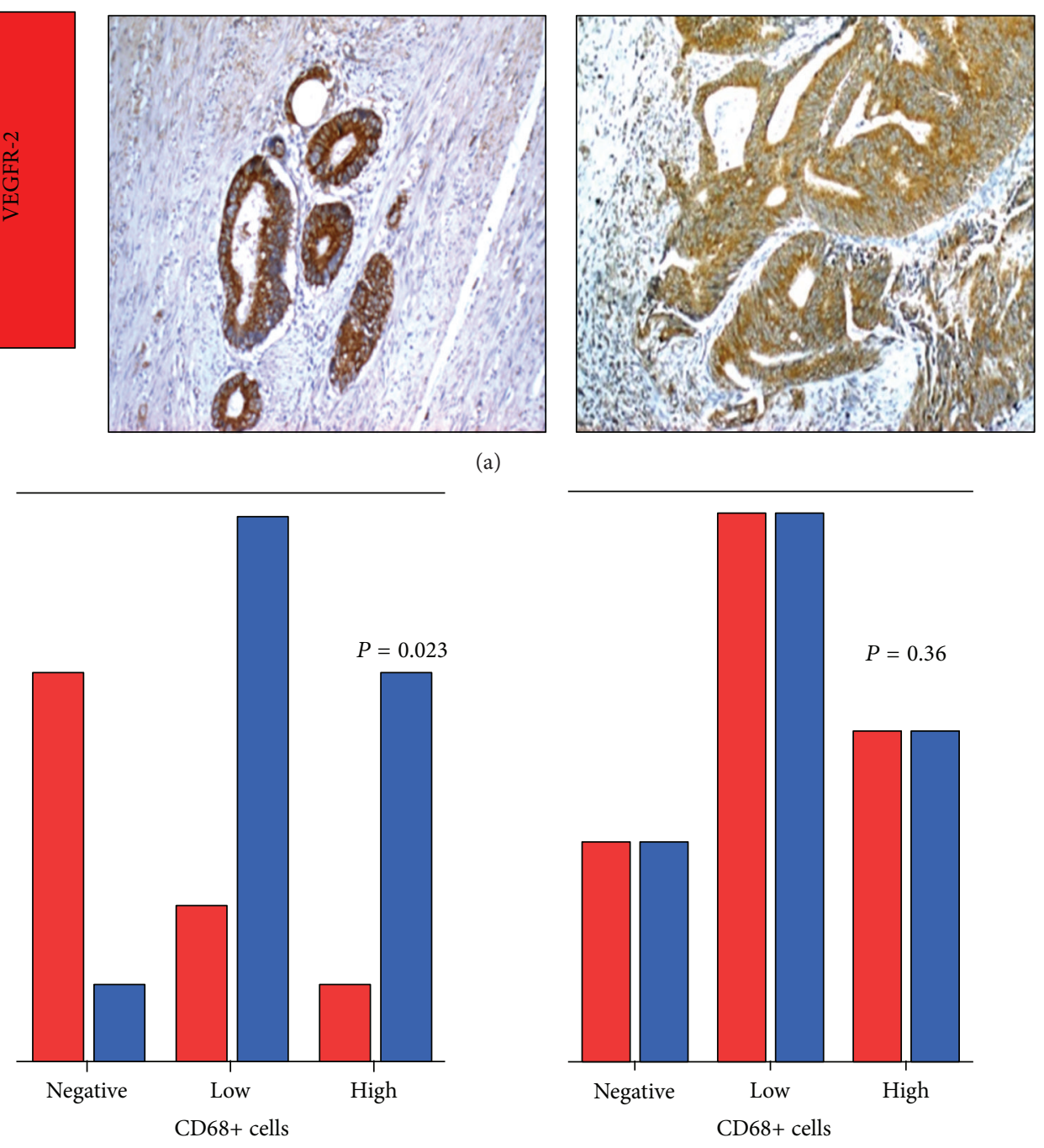

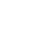

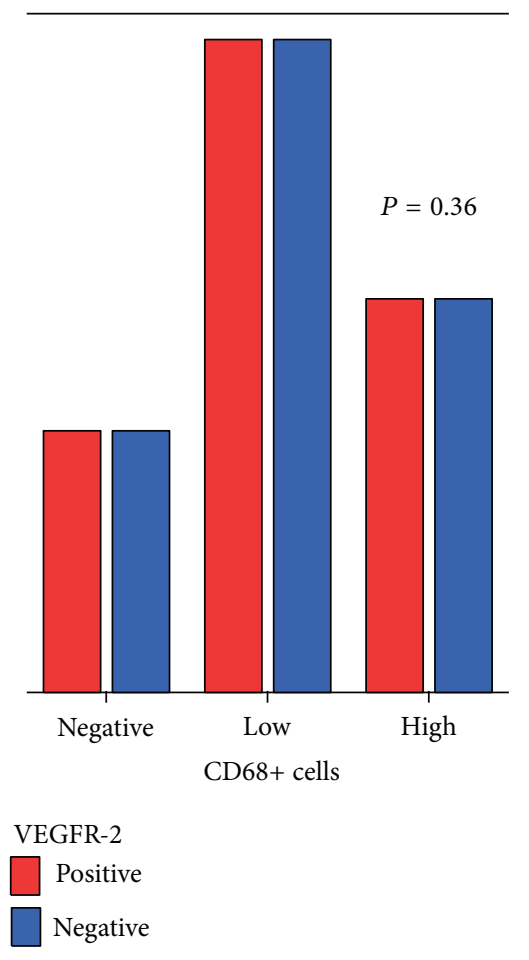

(b)

Figure 3: Continued. 

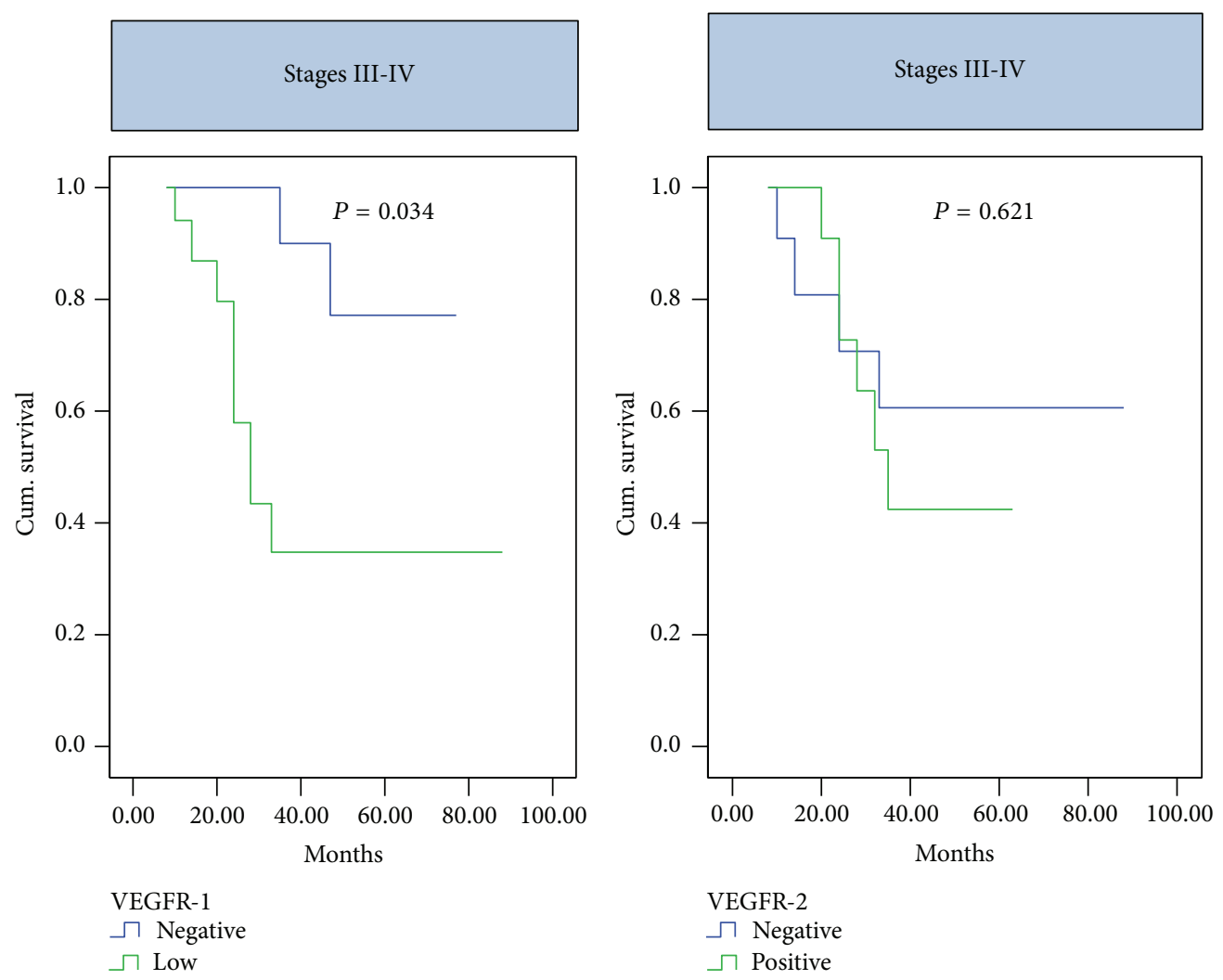

(c)

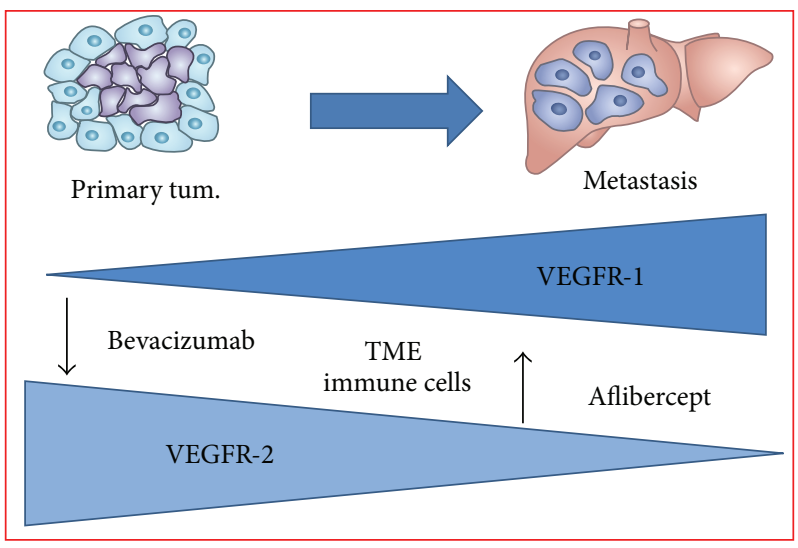

(d)

FIGURE 3: VEGFR-1 and VEGFR-2 differentially orchestrate the tumour-stroma interplay to promote cancer growth and metastasis. (a) Representative images of VEGFR-1 and VEGFR-2 immunostaining in a primary colon carcinoma and corresponding liver metastasis obtained from our still unpublished observations. Yellow and black arrows indicate the immunostaining in the stromal compartment and malignant colonic cells, respectively; with magnification 10x. (b) VEGFR-1 and VEGFR-2 immunostaining differentially correlate with CD68 infiltration, a marker of M2 tumor associate macrophages (TAMs). (c) Patients' disease specific survival in relation to VEGFR-1 and VEGFR-2 expression in our cohort of 86 CRC patients stage III-IV only (Pancione et al. unpublished data). (d) Schematic drawing of the proposed mechanism(s) involved in metastasis-promoting actions of VEGFR-1 and VEGFR-2, respectively. VEGFR-1, but not VEGFR-2, is expressed in a variety of stromal cells and appears synergized with TME in the evolution of premetastatic niche and cancer cell migration. Aflibercept targeting PlGF/VEGFR-1 axis can reduce the induction of an angiogenic rescue program and inhibit immune cells recruitment and metastatic progression. The $P$ value is reported in each graph. Cum. (cumulative), Met. (metastases), Neg. (negative), Pos. (positive), and Tum. (tumor). 
TABLE 1: Bevacizumab, aflibercept, and regorafenib are antiangiogenic drugs currently approved for mCRC treatment. These drugs have proved successful for the clinical treatment of various types of cancer and their mechanism of action results different affecting a variable range of cell types and signaling within tumor microenvironment.

\begin{tabular}{lll}
\hline Drug & Indication & Mechanism of action \\
\hline Bevacizumab & First-/second-line mCRC plus CT & MoAb binding VEGF-A \\
Aflibercept & Second-line mCRC plus FOLFIRI & VEGF decoy binding VEGF-A, VEGF-B, and PlGF \\
Regorafenib & Third-line mCRC as single agent & Multikinase inhibitor of RTKI of VEGFR-2 and -3, TIE-2, \\
\end{tabular}

PFS, and OS [71]. Recently, a meta-analysis of six randomized phase III studies showed that combination of bevacizumab to standard chemotherapy improves RR, PFS, and OS [72]. Bevacizumab main toxicities are peculiar and class specific and they are represented by arterial hypertension, bleeding, proteinuria, arterial or venous thrombosis, gastrointestinal perforation, and wound healing problems [70-72]. Another aspect of antiangiogenic treatment was recently investigated regarding the use of bevacizumab in patients who have progressed to a bevacizumab containing first-line therapy. The phase III randomized trial ML18147 evaluated the role of continuing bevacizumab plus standard chemotherapy after progression to bevacizumab plus chemotherapy first-line treatment. In this trial, patients who have received first-line bevacizumab containing therapy were randomized to receive second-line chemotherapy with alternate cytotoxic regimen with or without bevacizumab. Results obtained with this study show an advantage of maintaining bevacizumab plus chemotherapy beyond first progression, using an alternative cytotoxic regimen with significative prolongation in OS (11.2 versus 9.8 months; HR $0.81 ; 95 \%$ CI $0.69-0.84 ; P=0.0062$ ) and PFS (5.7 versus 4.1 months; HR 0.68; 95\% CI 0.59-0.78; $P<0.0001)$ [73].

4.2. Regorafenib: Mechanism of Action and Clinical Studies. Regorafenib is an oral multikinase inhibitor with activity against selected tyrosine kinases (VEGFR-2 and -3, TIE2, PDGFR, FGFR, RET, and c-Kit) as well as a signal transduction inhibitor of the RAF/MEK/ERK pathway (Figure 2 and Table 1) [74]. Regorafenib has been investigated as single agent in mCRC treatment after failure of standard therapy. The international, multicentered, randomized, double-blinded, placebo-controlled phase III trial CORRECT was conducted to evaluate efficacy of regorafenib in mCRC. In this study, 760 enrolled patients, who have progressed to all standard approved therapies, were randomized to receive regorafenib plus best supportive care (BSC) or placebo plus BSC. Primary end point of this study was OS. Preplanned interim analysis data showed a significantly longer OS in the regorafenib plus BSC arm than in the placebo plus BSC one (6.4 versus 5.0 months; HR 0.77; 95\% CI 0.64-0.94; $P=0.0052$ ). Also PFS was significantly prolonged by regorafenib (1.9 versus 1.7 months; HR 0.49; $P<0.000001)$. Response rate had no significative difference between two study arms ( $1 \%$ versus $0.4 \%$ ), but regorafenib arm resulted in a higher disease control rate compared to placebo ( $45 \%$ versus 15\%). Main toxicities related to this agent were represented by hand-foot skin reaction, fatigue, diarrhea, hypertension, and rash or desquamation [14].

\section{Aflibercept: Trap-Technology and Peculiar Mechanism of Action}

Aflibercept is a novel humanized recombinant fusion protein which acts as a decoy receptor binding to VEGF-A, VEGF$\mathrm{B}$, and PlGF resulting in the inhibition of their interaction with specific receptors (Figure 2 and Table 1). This molecule has been developed by employing the "trap technology" [75]. Aflibercept is a $97 \mathrm{kDA}$ homodimeric glycoprotein deriving from the fusion of VEGFR-1 and VEGFR-2 extracellular domains to Fc portion of human IgG1 [76-78]. Aflibercept binds VEGF-A with high affinity with a $0.49 \mathrm{pMKd}$. In this way, aflibercept prevents interactions between VEGF and receptors exposed on cellular surface. Interestingly, aflibercept shows also high affinity for PlGF-2 with a Kd of 39 pM. Different from other antiangiogenic drugs, this mechanism of action should result in inhibition of MDSCs known to promote tumor progression and antagonize the antitumor efficacy (Figure 2). Anti-PlGF activity enhancing VEGFR inhibitor therapy should reduce the release of angiogenic factors by tumour and vascular cells (Figure 2). Aflibercept does not cause as much hypoxia and therefore does not induce an angiogenic escape programme as strongly as VEGF-VEGFR inhibitors do. This peculiar mechanism of action might reduce the incidence of drug resistance and explain, at least in part, the indication of aflibercept as valid second-line therapeutic option (Figure 3(d)).

5.1. Preclinical Studies. In vitro assays on cell lines evidenced that aflibercept blocks even VEGFR-2 mediated phosphorylation resulting in inhibition of endothelial cells proliferation necessary for new blood vessels formation [79]. Aflibercept significantly inhibits tumor growth and angiogenesis, reduces tumor vessel density, and inhibits metastases in xenografts of various tumor types [80-82]. When combined, in tumor xenografts, with other anticancer treatments such as cytotoxic chemotherapy or radiotherapy, aflibercept shows greater inhibition of tumor growth and vasculature than with the individual treatments alone. In particular, aflibercept has been investigated alone and in combination with cytotoxic chemotherapy in several murine models studies. In a 3-arm mammary adenocarcinoma study, aflibercept $40 \mathrm{mg} / \mathrm{kg} /$ dose twice weekly was as active as the highest nontoxic dose of $5-\mathrm{FU}(90 \mathrm{mg} / \mathrm{kg} / \mathrm{dose})$ and the combination of two drugs showed synergistic activity at all tested doses. Single-agent aflibercept and irinotecan were equally active in mice with advanced-stage human colon cancer, with the combination of demonstrating synergy [83, 84]. Moreover, in tumor xenografts, aflibercept has shown 
decrease both in the expression of tumor vascular genes and in the activation of the vascular endothelial signalling pathways [85]. A dose-dependent effect has been observed in a xenograft model of neuroblastoma, whereas high doses of aflibercept led to greater regression of coopted vascular structures, which occurs during the initial phase of tumor growth [86]. Aflibercept has also shown activity on animal models in combination with several cytotoxic drugs such as docetaxel, paclitaxel, and gemcitabine or in combination with radiotherapy. It also showed synergistic activity with oxaliplatin, cisplatin, 5-fluorouracil, and S-1 [87-90]. Aflibercept activity has been observed at a dose ranging from $2.5 \mathrm{mg} / \mathrm{kg}$ to $40 \mathrm{mg} / \mathrm{kg}$.

5.2. Phases I-II Clinical Trials. A phase I dose-escalation study of i.v. aflibercept in combination with irinotecan, infusional fluorouracil, and leucovorin showed that the recommended dose of aflibercept is $4 \mathrm{mg} / \mathrm{kg}$ i.v. every 2 weeks. This was an open-label, sequential cohort, and doseescalation study. Primary end point was to find the dose limiting toxicity (DLT) during the first two cycles of treatment. Patients were treated with i.v. aflibercept over 1 hour on day 1 immediately followed by i.v. irinotecan $180 \mathrm{mg} / \mathrm{m}^{2}$ over 1 hour, then leucovorin $200 \mathrm{mg} / \mathrm{m}^{2}$ (or L-leucovorin $100 \mathrm{mg} / \mathrm{m}^{2}$ ) over 2 hours and 5-fluorouracil $400 \mathrm{mg} / \mathrm{m}^{2}$ i.v. bolus, and then $600 \mathrm{mg} / \mathrm{m}^{2}$ infusion over 22 hours on days 1 and 2 (Table 2). The first level dose of aflibercept was $2 \mathrm{mg} / \mathrm{kg}$, and patients who did not experiment toxicities were enrolled to higher dose levels $(4,5$, and $6 \mathrm{mg} / \mathrm{kg}$ ). Thirty-eight patients affected by different type of cancer were enrolled. $61 \%$ of patients had mCRC. 36 had received previous chemotherapy, with $63 \%$ having previously received irinotecan. Adverse events associated with VEGF blockade were represented by mild to moderate hypertension (74\%), dysphonia (74\%), epistaxis (58\%), and proteinuria (87\%). Free aflibercept blood concentrations exceeded VEGF-bound aflibercept ones throughout the dose interval starting from $4 \mathrm{mg} / \mathrm{kg}$. This observation suggested that aflibercept was administered at a biologically active dose [91]. Another phase I study investigated the safety dose limiting toxicities (DLTs) and recommended dose in 16 mCRC patients (Table 2). Two dose levels of aflibercept were assessed, $2 \mathrm{mg} / \mathrm{kg}$ and $4 \mathrm{mg} / \mathrm{kg}$, respectively. DLTs had to be evaluated in the first two cycles. No DLTs were found. At recommended dose of $4 \mathrm{mg} / \mathrm{kg}$, most common grade $3 / 4$ toxicities were neutropenia $75 \%$ for both doses and hypertension in $25 \%$ at $4.0 \mathrm{mg} / \mathrm{kg}$. The response rate and progression-free survival at $4.0 \mathrm{mg} / \mathrm{kg}$ were $8.3 \%$ and 7.59 months, respectively [92]. Aflibercept as monotherapy was investigated in two phase II trials (Table 2). In a phase II trial, 51 patients with mCRC who had received 1 or more lines of therapy were treated with aflibercept $4 \mathrm{mg} / \mathrm{kg}$ administered every 2 weeks. Primary end points were RR and 4 months PFS. 27 of 51 enrolled patients had received bevacizumab in the previous treatment regimens, while the remaining group was bevacizumab naïve. Disease control rate, defined as partial responses plus stable diseases, was $30 \%$ and $29 \%$, while PFS was 3.4 and 2.0 months in bevacizumab pretreated and bevacizumab naïve patients, respectively. 7 patients in each group maintained 4-month PFS [93]. A more recent two-stage phase II study evaluated $75 \mathrm{mCRC}$ patients treated with single-agent aflibercept at the dose of $4 \mathrm{mg} / \mathrm{kg}$ i.v. every 2 weeks. Patients were enrolled in two cohorts: bevacizumab naïve (24 patients) and prior bevacizumab (51 patients). Primary end point of this study was a combination of RR and 16-week PFS. The median PFS in the bevacizumab naive and prior bevacizumab groups was 2.0 and 2.4 months, respectively. Aflibercept showed limited activity as a single agent ant it was well tolerated in pretreated $\mathrm{mCRC}$, independent from prior therapy with bevacizumab [94]. Differently, the randomized phase 2 study AFFIRM investigated aflibercept combined with modified FOLFOX6 in first-line treatment of mCRC (Table 2). The primary end point was PFS rate at 12 months. The secondary end points included overall response rate, PFS, and OS. Preliminary results show no significant difference in PFS at 1 year [95].

5.3. The VELOUR Phase III Trial. The international, doubleblinded, phase III trial VELOUR randomized 1,226 patients to receive either aflibercept $4 \mathrm{mg} / \mathrm{kg}$ i.v in combination with FOLFIRI or placebo every 2 weeks in combination with FOLFIRI after oxaliplatin treatment failure. Patients had adequate organ function and Eastern Cooperative Oncology Group performance status was 0 to 2 . Median age was 61 years, $58.6 \%$ were men, and $56.4 \%$ had multiple metastatic sites. Approximately one-third of patients had previously been treated with bevacizumab. Primary end point was OS and secondary end points were PFS, RR, and also pharmacokinetics and immunogenicity. Addition of aflibercept to FOLFIRI significantly improved OS if compared to placebo plus FOLFIRI arm (12.06 versus 13.5 months; HR 0.817; 95.34\% CI 0.713-0.937; $P=0.0032$ ). PFS and response rate were also significantly improved. Median PFS was 6.90 months in the aflibercept arm and 4.67 months in the placebo arm (HR 0.758; 95\% CI 0.661-0.869; $P=0.0001$ ). Response rate was $19.8 \%$ (95\% CI 16.4-23.2\%) with aflibercept plus FOLFIRI versus $11.1 \%$ (95\% CI 8.5-13.8\%) with placebo plus FOLFIRI $(P=0.0001)$. Grades 3 and 4 adverse events with at least $2 \%$ higher incidence in aflibercept arm were represented by diarrhea, hypertension, asthenic conditions, neutropenia, proteinuria, stomatitis, ulceration, infections, gastrointestinal and abdominal pain, neutropenia, and neutropenic complications. Treatment discontinuation for toxicity occurred in $26.8 \%$ of patients receiving aflibercept plus FOLFIRI and $12.1 \%$ of patients receiving placebo [13]. A subset analysis evaluated differences in terms of outcome between patients who have progressed to an oxaliplatin plus bevacizumab therapy versus no prior bevacizumab treatment. This subgroups analysis found that the use of bevacizumab as a part of first line of therapy did not negatively affect clinical benefit of adding aflibercept to FOLFIRI [98] (Table 2). Given the results of VELOUR trial, aflibercept has been indicated for mCRC second-line treatment in addition to FOLFIRI in patients who have progressed to an oxaliplatin based first-line therapy independent from prior use of bevacizumab. 


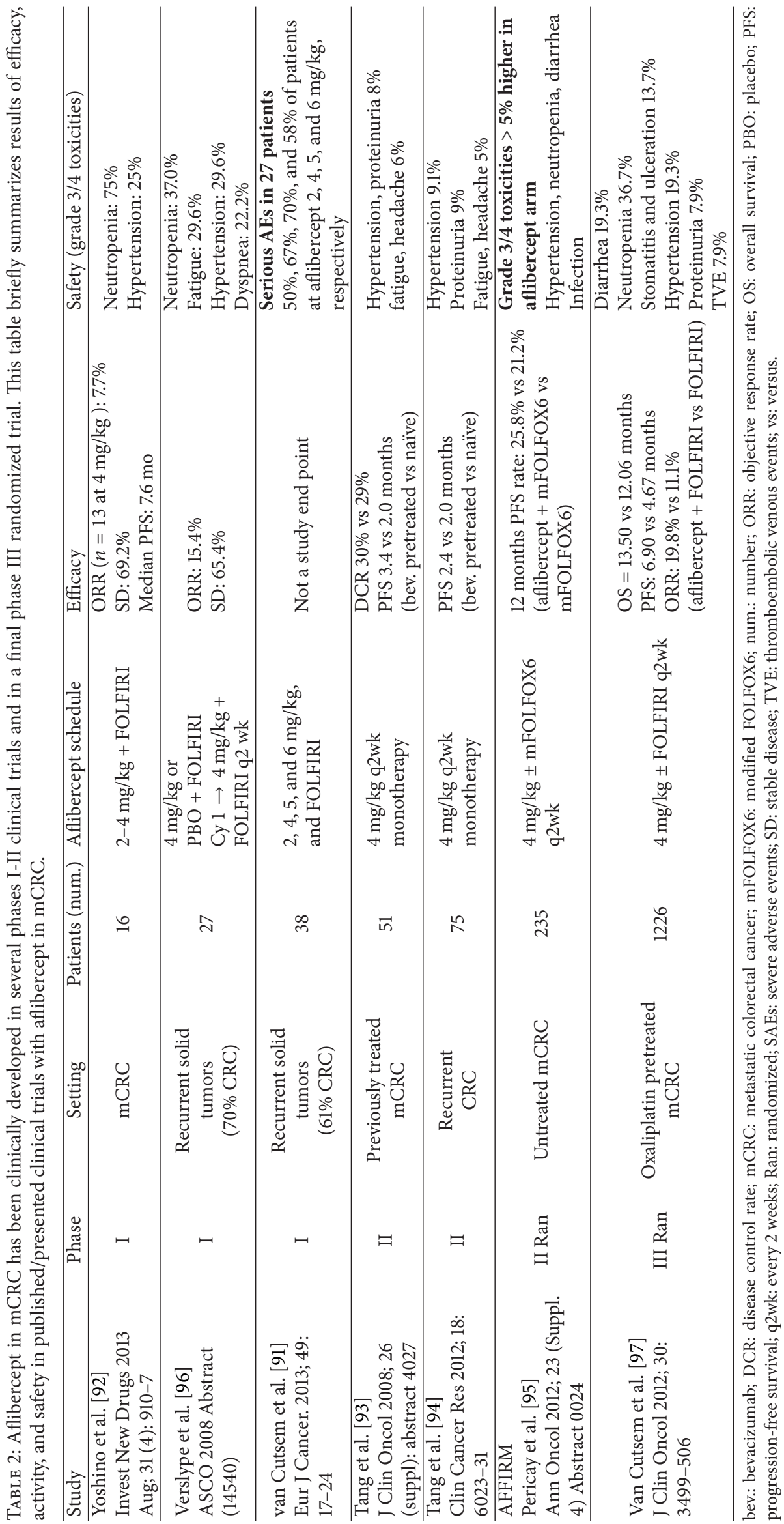




\section{Concluding Remarks}

Target agents have improved results obtained by standard chemotherapy in mCRC treatment and they have also enriched oncologists therapeutic weapons. Unfortunately, despite of these benefits, the prognosis of mCRC still remains poor as many patients experience disease progression after chemo- and target therapies. Today, the determination of Ras mutational status is mandatory before choosing the optimal strategy for treatment. Ras mutant status suggests that using anti-EGFR agents in these patients is not only ineffective but, in some cases, could be detrimental. On the other hand, antiangiogenic therapies could be proposed to every patient according to his performance status, comorbidities, and clinical conditions, because of the lack of biomarkers and predictive factors of response which may help us to better select patients for treatment with these molecules. Antiangiogenic agent bevacizumab has been approved in both first- and second-line treatment of mCRC. Clinical trials suggest that antiangiogenic treatment with bevacizumab could be continued also beyond first progression, and newer agents such as regorafenib and aflibercept have recently been approved in mCRC third- and second-line therapy, respectively. This evidence enforces the concept that targeting CRC through angiogenesis inhibition also after failure of a first antiangiogenic treatment could be equally successful. An important question to point out is about the optimal antiangiogenic strategy to use after a bevacizumab based first-line therapy. In this scenario, aflibercept seems to be an optimal molecule to target angiogenesis. Addition of aflibercept to FOLFIRI in second-line treatment for mCRC allowed a 1.4-month gain in OS that is identic to OS prolongation reached by bevacizumab in second-line treatment in ML18147 study. Given toxicity profile and not significantly higher survival benefit of aflibercept in second-line therapy if compared with bevacizumab beyond first progression, one could conclude that aflibercept is not an ideal agent for this setting. Nevertheless, aflibercept has a peculiar and unique mechanism of action that is different from other antiangiogenic agents. In fact, whereas bevacizumab targets angiogenesis through VEGF-A inhibition, aflibercept inhibits VEGF-A, -B and PlGF signaling pathways. This mechanism of action should allow the blockage of tumor progression at different levels acting on malignant cells, macrophages, and stromal cells that contribute with cooperative mechanisms to tumour neovascularization. Therefore, using aflibercept in second-line therapy in patients who have progressed to bevacizumab may help (1) to overcome resistances, (2) limit the compensatory angiogenic factors, and (3) modulate the activity of inflammatory cells within the tumor microenvironment. This concept is consistent with clinical evidences that VEGF-A inhibition, in bevacizumab treated patients, is associated with VEGFR-2 and PlGF increased levels. In this way, PlGF contributes to the induction of an angiogenic rescue program while VEGF-A is blocked, thus contributing to tumor escape to bevacizumab action. This suggests that aflibercept could be active in avoiding tumor escape mechanism and restore sensitivity to antiangiogenic treatment (Figure 3(d)). Moreover, given the crucial role of PlGF/VEGFR-1 axis in inflammation, neovascularization, and tumor aggressiveness, the use of aflibercept could be helpful to target both proangiogenic factors and the inflammatory cells surrounding the tumor microenvironment.

Aflibercept has improved OS, PFS, and RR in secondline therapy for $\mathrm{mCRC}$ in addition to FOLFIRI regimen after failure of a first-line oxaliplatin containing regimen. Actually, this new molecule can be considered a standard of care in this setting both in Ras wild-type and mutant patients and it can be successfully used after anti-EGFR molecules as well as after bevacizumab treatment. Its peculiar mechanism of action may help to overcome tumor escape mechanisms to bevacizumab treatment. Nowadays, we have no biomarkers of response to aflibercept that may help us to candidate patient to this therapy. Further studies are required to better investigate the role of aflibercept in the contest of a global strategy of treatment for mCRC.

\section{Conflict of Interests}

All the authors declare that they have no conflict of interests.

\section{References}

[1] R. Siegel, E. Ward, O. Brawley, and A. Jemal, "Cancer statistics," CA: Cancer Journal for Clinicians, vol. 61, no. 4, pp. 212-236, 2011.

[2] A. Jemal, F. Bray, M. M. Center, J. Ferlay, E. Ward, and D. Forman, "Global cancer statistics," CA Cancer Journal for Clinicians, vol. 61, no. 2, pp. 69-90, 2011.

[3] J. Y. Douillard, D. Cunningham, A. D. Roth et al., "Irinotecan combined with fluorouracil compared with fluorouracil alone as first-line treatment for metastatic colorectal cancer: a multicentre randomised trial," The Lancet, vol. 355, no. 9209, pp. 1041$1047,2000$.

[4] L. B. Saltz, J. V. Cox, C. Blanke et al., "Irinotecan plus fluorouracil and leucovorin for metastatic colorectal cancer," The New England Journal of Medicine, vol. 343, no. 13, pp. 905-914, 2000.

[5] R. M. Goldberg, D. J. Sargent, R. F. Morton et al., "A randomized controlled trial of fluorouracil plus leucovorin, irinotecan, and oxaliplatin combinations in patients with previously untreated metastatic colorectal cancer," Journal of Clinical Oncology, vol. 22, no. 1, pp. 23-30, 2004.

[6] A. Grothey and D. Sargent, "Overall survival of patients with advanced colorectal cancer correlates with availability of fluorouracil, irinotecan, and oxaliplatin regardless of whether doublet or single-agent therapy is used first line," Journal of Clinical Oncology, vol. 23, no. 36, pp. 9441-9442, 2005.

[7] Y. S. R. Krishnaiah and M. A. Khan, "Strategies of targeting oral drug delivery systems to the colon and their potential use for the treatment of colorectal cancer," Pharmaceutical Development and Technology, vol. 17, no. 5, pp. 521-540, 2012.

[8] C. Bokemeyer, E. Van Cutsem, P. Rougier et al., "Addition of cetuximab to chemotherapy as first-line treatment for KRAS wild-type metastatic colorectal cancer: pooled analysis of the CRYSTAL and OPUS randomised clinical trials," European Journal of Cancer, vol. 48, no. 10, pp. 1466-1475, 2012.

[9] G. Argiles, R. Dienstmann, E. Elez, and J. Tabernero, "Panitumumab: a summary of clinical development in colorectal cancer 
and future directions," Future Oncology, vol. 8, no. 4, pp. 373389, 2012.

[10] J. Y. Douillard, K. S. Oliner, and S. Siena, "PanitumumabFOLFOX4 treatment and RAS mutations in colorectal cancer," The New England Journal of Medicine, vol. 369, no. 11, pp. 10231034, 2013.

[11] J. Folkman, “Tumor angiogenesis: therapeutic implications.," The New England Journal of Medicine, vol. 285, no. 21, pp. 11821186,1971

[12] H. Hurwitz, L. Fehrenbacher, W. Novotny et al., "Bevacizumab plus irinotecan, fluorouracil, and leucovorin for metastatic colorectal cancer," The New England Journal of Medicine, vol. 350, no. 23, pp. 2335-2342, 2004.

[13] E. van Cutsem, J. Tabernero, R. Lakomy et al., "Addition of aflibercept to fluorouracil, leucovorin, and irinotecan improves survival in a phase III randomized trial in patients with metastatic colorectal cancer previously treated with an oxaliplatin-based regimen," Journal of Clinical Oncology, vol. 30, no. 28, pp. 3499-3506, 2012.

[14] A. Grothey, E. Van Cutsem, A. Sobrero et al., "Regorafenib monotherapy for previously treated metastatic colorectal cancer (CORRECT): an international, multicentre, randomised, placebo-controlled, phase 3 trial," The Lancet, vol. 381, no. 9863 , pp. 303-312, 2013.

[15] J. A. Joyce and J. W. Pollard, "Microenvironmental regulation of metastasis," Nature Reviews Cancer, vol. 9, no. 4, pp. 239-252, 2009.

[16] M. H. McLean, G. I. Murray, K. N. Stewart et al., "The inflammatory microenvironment in colorectal neoplasia," PLoS ONE, vol. 6, no. 1, Article ID e15366, 2011.

[17] I. P. Witz, "The tumor microenvironment: the making of a paradigm," Cancer Microenvironment, vol. 2, supplement 1, pp. 9-17, 2009.

[18] D. I. Gabrilovich and S. Nagaraj, "Myeloid-derived suppressor cells as regulators of the immune system," Nature Reviews Immunology, vol. 9, no. 3, pp. 162-174, 2009.

[19] C. Fischer, M. Mazzone, B. Jonckx, and P. Carmeliet, "FLT1 and its ligands VEGFB and PlGF: drug targets for anti-angiogenic therapy?" Nature Reviews Cancer, vol. 8, no. 12, pp. 942-956, 2008.

[20] J. D. Schwartz, E. K. Rowinsky, H. Youssoufian, B. Pytowski, and $\mathrm{Y}$. Wu, "Vascular endothelial growth factor receptor-1 in human cancer: concise review and rationale for development of IMC18F1 (Human antibody targeting vascular endothelial growth factor receptor-1)," Cancer, vol. 116, no. 4, pp. 1027-1032, 2010.

[21] D. I. Gabrilovich, S. Ostrand-Rosenberg, and V. Bronte, "Coordinated regulation of myeloid cells by tumours," Nature Reviews Immunology, vol. 12, no. 4, pp. 253-268, 2012.

[22] A. Mantovani, A. Sica, P. Allavena, C. Garlanda, and M. Locati, "Tumor-associated macrophages and the related myeloidderived suppressor cells as a paradigm of the diversity of macrophage activation," Human Immunology, vol. 70, no. 5, pp. 325-330, 2009.

[23] M. L. Squadrito and M. de Palma, "Macrophage regulation of tumor angiogenesis: implications for cancer therapy," Molecular Aspects of Medicine, vol. 32, no. 2, pp. 123-145, 2011.

[24] J. Wels, R. N. Kaplan, S. Rafii, and D. Lyden, "Migratory neighbors and distant invaders: tumor-associated niche cells," Genes and Development, vol. 22, no. 5, pp. 559-574, 2008.

[25] J. Folkman, E. Merler, C. Abernathy, and G. Williams, "Isolation of a tumor factor responsible or angiogenesis," The Journal of Experimental Medicine, vol. 133, no. 2, pp. 275-288, 1971.
[26] J. Folkman, "Role of angiogenesis in tumor growth and metastasis," Seminars in Oncology, vol. 29, 6, supplement 6, pp. 15-18, 2002.

[27] J. Folkman, "Fundamental concepts of the angiogenic process," Current Molecular Medicine, vol. 3, no. 7, pp. 643-651, 2003.

[28] P. Carmeliet, "Angiogenesis in life, disease and medicine," Nature, vol. 438, no. 7070, pp. 932-936, 2005.

[29] N. Ferrara, "Vascular endothelial growth factor: basic science and clinical progress," Endocrine Reviews, vol. 25, no. 4, pp. 581611, 2004.

[30] D. Shweiki, M. Neeman, A. Itin, and E. Keshet, "Induction of vascular endothelial growth factor expression by hypoxia and by glucose deficiency in multicell spheroids: implications for tumor angiogenesis," Proceedings of the National Academy of Sciences of the United States of America, vol. 92, no. 3, pp. 768$772,1995$.

[31] D. J. Hicklin and L. M. Ellis, "Role of the vascular endothelial growth factor pathway in tumor growth and angiogenesis," Journal of Clinical Oncology, vol. 23, no. 5, pp. 1011-1027, 2005.

[32] G. Neufeld, T. Cohen, S. Gengrinovitch, and Z. Poltorak, "Vascular endothelial growth factor (VEGF) and its receptors," FASEB Journal, vol. 13, no. 1, pp. 9-22, 1999.

[33] G. D. Yancopoulos, S. Davis, N. W. Gale, J. S. Rudge, S. J. Wiegand, and J. Holash, "Vascular-specific growth factors and blood vessel formation," Nature, vol. 407, no. 6801, pp. 242-248, 2000.

[34] M. Shibuya, "Tyrosine kinase receptor Flt/VEGFR family: its characterization related to angiogenesis and cancer," Genes and Cancer, vol. 1, no. 11, pp. 1119-1123, 2011.

[35] L. Claesson-Welsh and M. Welsh, "VEGFA and tumour angiogenesis," Journal of Internal Medicine, vol. 273, no. 2, pp. 114-127, 2013.

[36] V. Joukov, K. Pajusola, A. Kaipainen et al., "A novel vascular endothelial growth factor, VEGF-C, is a ligand for the Flt4 (VEGFR-3) and KDR (VEGFR-2) receptor tyrosine kinases," The EMBO Journal, vol. 15, no. 2, pp. 290-298, 1996.

[37] K. Holmqvist, M. J. Cross, C. Rolny et al., “The adaptor protein Shb binds to tyrosine 1175 in vascular endothelial growth factor (VEGF) receptor-2 and regulates VEGF-dependent cellular migration," The Journal of Biological Chemistry, vol. 279, no. 21, pp. 22267-22275, 2004.

[38] F. Zhang, Z. Tang, and X. Hou, "VEGF-B is indispensable for blood vessel growth but critical for their survival, and VEGF-B targeting inhibits pathological angiogenesis," Proceedings of the National Academy of Sciences of the United States of America, vol. 106, pp. 6152-6157, 2009.

[39] L. Jussila and K. Alitalo, "Vascular growth factors and lymphangiogenesis," Physiological Reviews, vol. 82, no. 3, pp. 673-700, 2002.

[40] A. Khaliq, X. F. Li, M. Shams et al., "Localisation of placenta growth factor (PIGF) in human term placenta," Growth Factors, vol. 13, no. 3-4, pp. 243-250, 1996.

[41] P. Vuorela, E. Hatva, A. Lymboussaki et al., "Expression of vascular endothelial growth factor and placenta growth factor in human placenta," Biology of Reproduction, vol. 56, no. 2, pp. 489-494, 1997.

[42] S. R. Brave, C. Eberlein, M. Shibuya, S. R. Wedge, and S. T. Barry, "Placental growth factor neutralising antibodies give limited anti-angiogenic effects in an in vitro organotypic angiogenesis model," Angiogenesis, vol. 13, no. 4, pp. 337-347, 2010. 
[43] J. E. Park, H. H. Chen, J. Winer, K. A. Houck, and N. Ferrara, "Placenta growth factor. Potentiation of vascular endothelial growth factor bioactivity, in vitro and in vivo, and high affinity binding to Flt-1 but not to Flk-1/KDR," The Journal of Biological Chemistry, vol. 269, no. 41, pp. 25646-25654, 1994.

[44] P. Carmeliet, L. Moons, A. Luttun et al., "Synergism between vascular endothelial growth factor and placental growth factor contributes to angiogenesis and plasma extravasation in pathological conditions," Nature Medicine, vol. 7, no. 5, pp. 575-583, 2001.

[45] S. F. Martins, E. A. Garcia, M. A. Luz, F. Pardal, M. Rodrigues, and A. L. Filho, "Clinicopathological correlation and prognostic significance of VEGF-A, VEGF-C, VEGFR-2 and VEGFR-3 expression in colorectal cancer," Cancer Genomics Proteomics, vol. 10, no. 2, pp. 55-67, 2013.

[46] T. Wang, Z. Chen, X. Wei, B. Wei, and W. Dong, "Serum vascular endothelial growth factor-C and lymphoangiogenesis are associated with the lymph node metastasis and prognosis of patients with colorectal cancer," ANZ Journal of Surgery, vol. 81, no. 10, pp. 694-699, 2011.

[47] A. A. Alabi, A. Suppiah, L. A. Madden, J. R. Monson, and J. Greenman, "Preoperative serum levels of serum is associated with distant metastasis in cancer," International Journal of Colorectal Disease, vol. 24, no. 3, pp. 269-274, 2009.

[48] F. J. Gonzalez, A. R. Quesada, I. Sevilla et al., "Prognostic value of serum angiogenic activity in colorectal cancer patients," Journal of Cellular and Molecular Medicine, vol. 11, no. 1, pp. 120128, 2007.

[49] D. Fukumura, D. G. Duda, L. L. Munn, and R. K. Jain, "Tumor microvasculature and microenvironment: novel insights through intravital imaging in pre-clinical models," Microcirculation, vol. 17, no. 3, pp. 206-225, 2010.

[50] V. Barresi, C. Di Gregorio, L. Regiani-Bonetti, M. Ponz-De Leon, G. Barresi, and E. Vitarelli, "Stage i colorectal carcinoma: VEGF immunohistochemical expression, microvessel density, and their correlation with clinical outcome," Virchows Archiv, vol. 457, no. 1, pp. 11-19, 2010.

[51] Y. Takahashi, S. L. Tucker, Y. Kitadai et al., "Vessel counts and expression of vascular endothelial growth factor as prognostic factors in node-negative colon cancer," Archives of Surgery, vol. 132, no. 5, pp. 541-546, 1997.

[52] J. C. Lee, N. H. Chow, S. T. Wang, and S. M. Huang, "Prognostic of vascular endothelial growth factor expression in cancer," European Journal of Cancer, vol. 36, no. 6, pp. 748-753, 2000.

[53] F. De Vita, M. Orditura, E. Lieto et al., "Elevated perioperative serum vascular endothelial growth factor levels in patients with colon carcinoma," Cancer, vol. 100, no. 2, pp. 270-278, 2004.

[54] S. Onogawa, Y. Kitadai, S. Tanaka, T. Kuwai, S. Kimura, and K. Chayama, "Expression of VEGF-C and VEGF-D at the invasive edge correlates with lymph node metastasis and prognosis of patients with colorectal carcinoma," Cancer Science, vol. 95, no. 1, pp. 32-39, 2004.

[55] A. Giatromanolaki, E. Sivridis, and M. I. Koukourakis, "Angiogenesis in colorectal cancer: prognostic and therapeutic implications," American Journal of Clinical Oncology: Cancer Clinical Trials, vol. 29, no. 4, pp. 408-417, 2006.

[56] J. Cheng, R. E. Slavin, J. A. Gallagher et al., "Expression of vascular endothelial growth factor and receptor flk-1 in colon cancer liver metastases," Journal of Hepato-Biliary-Pancreatic Surgery, vol. 11, no. 3, pp. 164-170, 2004.

[57] M. Eppenberger, I. Zlobec, D. Baumhoer, L. Terracciano, and A. Lugli, "Role of the VEGF ligand to receptor ratio in the progression of mismatch repair-proficient colorectal cancer," BMC Cancer, vol. 11, no. 1, p. 93, 2010.

[58] Y. J. Lee, D. L. Karl, U. N. Maduekwe et al., "Differential effects of VEGFR-1 and VEGFR-2 inhibition on tumor metastases based on host organ environment," Cancer Research, vol. 70, no. 21, pp. 8357-8367, 2010.

[59] T. Tokunaga, Y. Oshika, Y. Abe et al., "Vascular endothelial growth factor (VEGF) mRNA isoform expression pattern is correlated with liver metastasis and poor prognosis in colon cancer," British Journal of Cancer, vol. 77, no. 6, pp. 998-1002, 1998.

[60] S. E. Duff, M. Jeziorska, D. D. Rosa et al., "Vascular endothelial growth factors and receptors in colorectal cancer: implications for anti-angiogenic therapy," European Journal of Cancer, vol. 42, no. 1, pp. 112-117, 2006.

[61] Y. Harada, Y. Ogata, and K. Shirouzu, "Expression of vascular endothelial growth factor and its receptor KDR (kinase domaincontaining receptor)/Flk-1 (fetal liver kinase-1) as prognostic factors in human colorectal cancer," International Journal of Clinical Oncology, vol. 6, no. 5, pp. 221-228, 2001.

[62] C. Eveno and M. Pocard, "VEGF levels and the angiogenic potential of the microenvironment can affect surgical strategy for colorectal liver metastasis," Cell Adhesion and Migration, vol. 6, no. 6, pp. 569-573, 2012.

[63] N. Beauchemin, "The colorectal tumor microenvironment: the next decade," Cancer Microenvironment, vol. 4, no. 2, pp. 181185, 2011.

[64] V. W. M. van Hinsbergh, A. Collen, and P. Koolwijk, "Angiogenesis and anti-angiogenesis: perspectives for the treatment of solid tumors," Annals of Oncology, vol. 10, supplement 4, pp. S60-S63, 1999.

[65] N. Ferrara and R. S. Kerbel, "Angiogenesis as a therapeutic target," Nature, vol. 438, no. 7070, pp. 967-974, 2005.

[66] A. Grothey and E. Galanis, "Targeting angiogenesis: progress with anti-VEGF treatment with large molecules," Nature Reviews Clinical Oncology, vol. 6, no. 9, pp. 507-518, 2009.

[67] H. Prenen, L. Vecchione, and E. van Cutsem, "Role of targeted agents in metastatic colorectal cancer," Targeted Oncology, vol. 8, no. 2, pp. 83-96, 2013.

[68] D. J. Cohen and H. S. Hochster, "Update on clinical data with regimens inhibiting angiogenesis and epidermal growth factor receptor for patients with newly diagnosed metastatic colorectal cancer," Clinical Colorectal Cancer, vol. 7, no. 1, pp. S21-S27, 2007.

[69] http://www.ema.europa.eu/ema/.

[70] J. Cassidy, S. Clarke, E. Díaz-Rubio et al., "XELOX vs FOLFOX4 as first-line therapy for metastatic colorectal cancer: NO16966 updated results," British Journal of Cancer, vol. 105, no. 1, pp. 58 64, 2011.

[71] B. J. Giantonio, P. J. Catalano, N. J. Meropol et al., "Bevacizumab in combination with oxaliplatin, fluorouracil, and leucovorin (FOLFOX4) for previously treated metastatic colorectal cancer: results from the Eastern Cooperative Oncology Group Study E3200," Journal of Clinical Oncology, vol. 25, no. 12, pp. 15391544, 2007.

[72] L. T. Macedo, A. B. da Costa Lima, and A. D. Sasse, "Addition of bevacizumab to first-line chemotherapy in advanced colorectal cancer: a systematic review and meta-analysis, with emphasis on chemotherapy subgroups," BMC Cancer, vol. 12, article 89, 2012.

[73] J. Bennouna, J. Sastre, D. Arnold et al., "Continuation of bevacizumab after first progression in metastatic colorectal 
cancer (ML18147): a randomised phase 3 trial," The Lancet Oncology, vol. 14, no. 1, pp. 29-37, 2013.

[74] S. L. Davis, S. G. Eckhardt, W. A. Messersmith, and A. Jimeno, "The development of regorafenib and its current and potential future role in cancer therapy," Drugs of Today, vol. 49, no. 2, pp. 105-115, 2013.

[75] A. N. Economides, L. R. Carpenter, J. S. Rudge et al., "Cytokine traps: multi-component, high-affinity blockers of cytokine action," Nature Medicine, vol. 9, no. 1, pp. 47-52, 2003.

[76] J. Holash, S. Davis, N. Papadopoulos et al., "VEGF-Trap: a VEGF blocker with potent antitumor effects," Proceedings of the National Academy of Sciences of the United States of America, vol. 99, no. 17, pp. 11393-11398, 2002.

[77] K. Jin, Y. Shen, K. He, Z. Xu, G. Li, and L. Teng, "Aflibercept (VEGF Trap): one more double-edged sword of anti-VEGF therapy for cancer?" Clinical and Translational Oncology, vol. 12, no. 8, pp. 526-532, 2010.

[78] N. Papadopoulos, J. Martin, Q. Ruan et al., "Binding and neutralization of vascular endothelial growth factor (VEGF) and related ligands by VEGF Trap, ranibizumab and bevacizumab," Angiogenesis, vol. 15, no. 2, pp. 171-185, 2012.

[79] A. T. Byrne, L. Ross, J. Holash et al., "Vascular endothelial growth factor-trap decreases tumor burden, inhibits ascites, and causes dramatic vascular remodeling in an ovarian cancer model," Clinical Cancer Research, vol. 9, no. 15, pp. 5721-5728, 2003.

[80] H. M. W. Verheul, H. Hammers, K. Van Erp et al., "Vascular endothelial growth factor trap blocks tumor growth, metastasis formation, and vascular leakage in an orthotopic murine renal cell cancer model," Clinical Cancer Research, vol. 13, no. 14, pp. 4201-4208, 2007.

[81] M. Chiron, P. Lejeune, and F. Blandt, "Broad spectrum of antitumor activity of aflibercept (VEGF-Trap) in tumor-bearing mice, Proc Am Assoc Cancer Res," in Proceedings of the American Association for Cancer Research, Abstract 380, 2008.

[82] M. Fukasawa and M. Korc, "Vascular endothelial growth factortrap suppresses tumorigenicity of multiple pancreatic cancer cell lines," Clinical Cancer Research, vol. 10, no. 10, pp. 3327-3332, 2004.

[83] P. R. Wachsberger, R. Burd, C. Cardi et al., "VEGF Trap in combination with radiotherapy improves tumor control in U87 glioblastoma," International Journal of Radiation Oncology, Biology, Physics, vol. 67, no. 5, pp. 1526-1537, 2007.

[84] M. V. P. Chiron, P. Lejeune, B. Demers, D. Leopold, and M. C. Bissery, "Synergistic activity of aflibercept (VEGF Trap) in combination with 5-fluorouracil and irinotecan in preclinical tumor models," in Proceeding from AACR-NCI-EORTC: Molecular Targets and Cancer Therapeutics, San Francisco, Calif, USA, Abstract A13, 2007.

[85] W. Lassoued, D. Murphy, J. Tsai, R. Oueslati, G. Thurston, and W. M. F. Lee, "Effect of VEGF and VEGF Trap on vascular endothelial cell signaling in tumors," Cancer Biology and Therapy, vol. 10, no. 12, pp. 1326-1333, 2010.

[86] E. S. Kim, A. Serur, J. Huang et al., "Potent VEGF blockade causes regression of coopted vessels in a model of neuroblastoma," Proceedings of the National Academy of Sciences of the United States of America, vol. 99, no. 17, pp. 11399-11404, 2002.

[87] M. Chiron, P. Vrignaud, and P. Lejeune, "in vivo evaluation of the angiogenic agent VEGF Trap," in Proceedings of the American Association for Cancer Research, Abstract 547, 2005.

[88] L. Hu, J. Hofmann, J. Holash, G. D. Yancopoulos, A. K. Sood, and R. B. Jaffe, "Vascular endothelial growth factor trap combined with paclitaxel strikingly inhibits tumor and ascites, prolonging survival in a human ovarian cancer model," Clinical Cancer Research, vol. 11, no. 19, pp. 6966-6971, 2005.

[89] P. Lejeune, M. Chiron, and R. Le Moigne, "Combination of the angiogenic agent aflibercept (VEGF-Trap) with docetaxel or gemcitabine results in greater antitumor activity in tumor bearing mice," in Proceedings of the American Association for Cancer Research, Abstract 1107, 2008.

[90] P. R. Wachsberger, R. Burd, C. Cardi et al., "VEGF trap in combination with radiotherapy improves tumor control in U87 glioblastoma," International Journal of Radiation Oncology, Biology, Physics, vol. 67, no. 5, pp. 1526-1537, 2007.

[91] E. van Cutsem, D. Khayat, C. Verslype et al., "Phase I doseescalation study of intravenous aflibercept administered in combination with irinotecan, 5-fluorouracil and leucovorin in patients with advanced solid tumours," European Journal of Cancer, vol. 49, no. 1, pp. 17-24, 2013.

[92] T. Yoshino, K. Yamazaki, K. Yamaguchi et al., "Phase I study of intravenous aflibercept with FOLFIRI in Japanese patients with previously treated metastatic colorectal cancer," Investigational New Drugs, vol. 31, no. 4, pp. 910-917, 2013.

[93] P. Tang, S. J. Cohen, G. A. Bjarnason et al., "Phase II trial of aflibercept (VEGF Trap) in previously treated patients with metastatic colorectal cancer (MCRC): a PMH phase II consortium trial," Journal of Clinical Oncology, vol. 26, supplement, 2008, abstract 4027.

[94] P. A. Tang, S. J. Cohen, C. Kollmannsberger et al., "Phase II clinical and pharmacokinetic study of aflibercept in patients with previously treated metastatic colorectal cancer," Clinical Cancer Research, vol. 18, no. 21, pp. 6023-6031, 2012.

[95] C. Pericay, G. Folprecht, M. Saunders et al., "Phase 2 randomized, noncomparative, open-label study of aflibercept and modified Folfox6 in the first-line treatment of metastatic colorectal cancer (AFFIRM)," Annals of Oncology, supplement 4, no. 23, p. iv16, 2012.

[96] Verslype et al., ASCO 2008 Abstract, 1450.

[97] E. Van Cutsem, J. Tabernero, R. Lakomy et al., "Addition of aflibercept to fluorouracil, leucovorin, and irinotecan improves survival in a phase III randomized trial in patients with metastatic colorectal cancer previously treated with an oxaliplatin-based regimen," Journal of Clinical Oncology, vol. 30, no. 28, pp. 3499-3506, 2012.

[98] C. J. Allegra, R. Lakomy, J. Tabernero et al., "Effects of prior bevacizumab (B) use on outcomes from the VELOUR study: A phase III study of aflibercept (Afl) and FOLFIRI in patients (pts) with metastatic colorectal cancer (mCRC) after failure of an oxaliplatin regimen," Journal of Clinical Oncology, no. 30, 2012, abstract 3505 . 


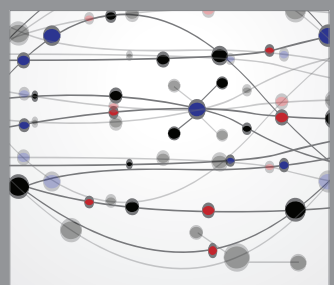

The Scientific World Journal
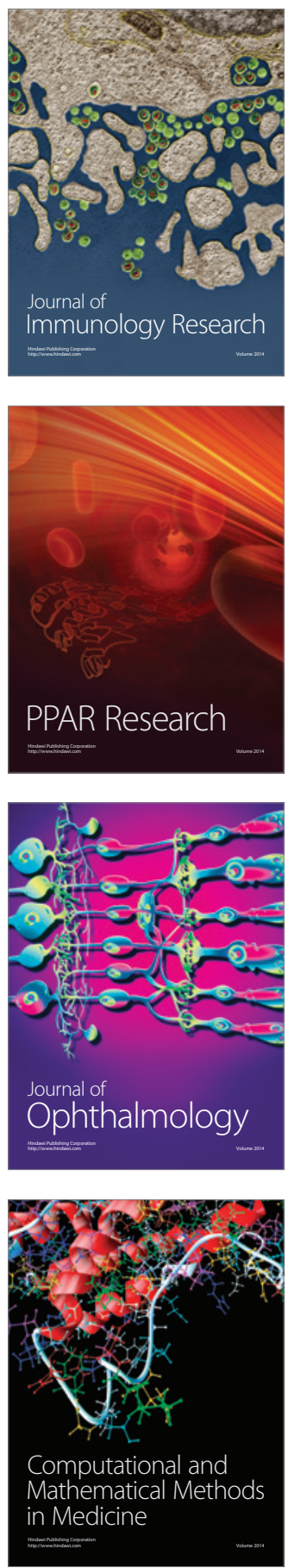

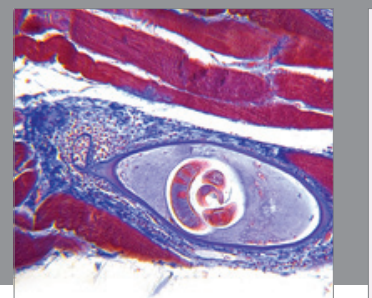

Gastroenterology

Research and Practice
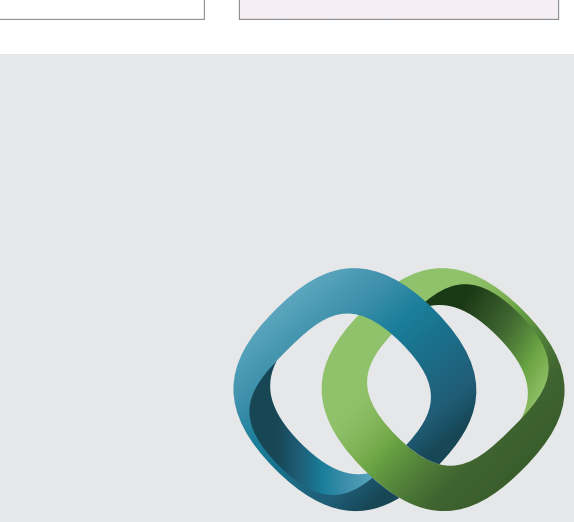

\section{Hindawi}

Submit your manuscripts at

http://www.hindawi.com
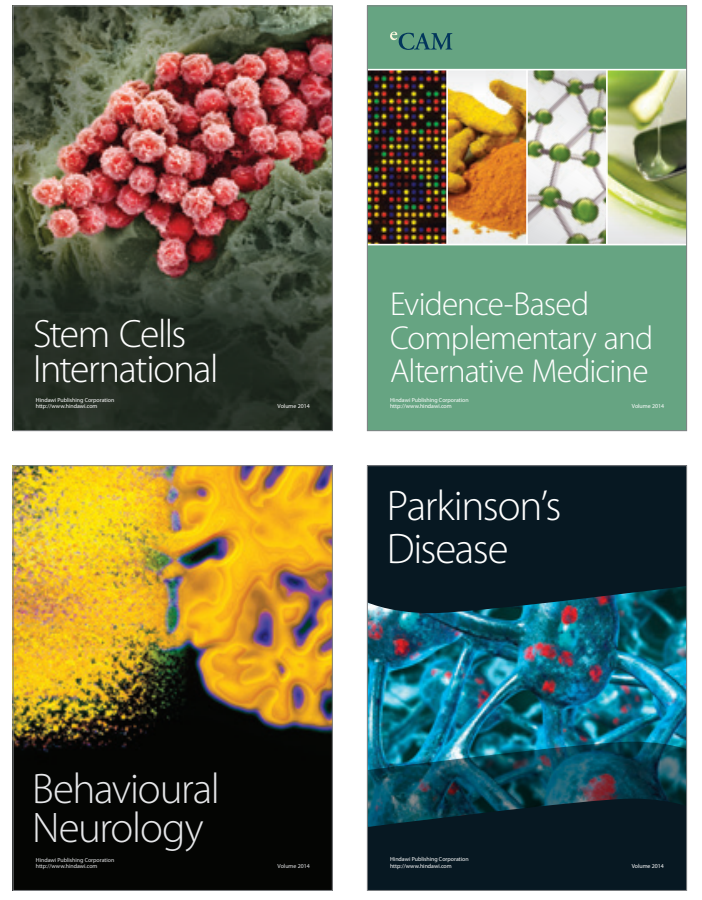
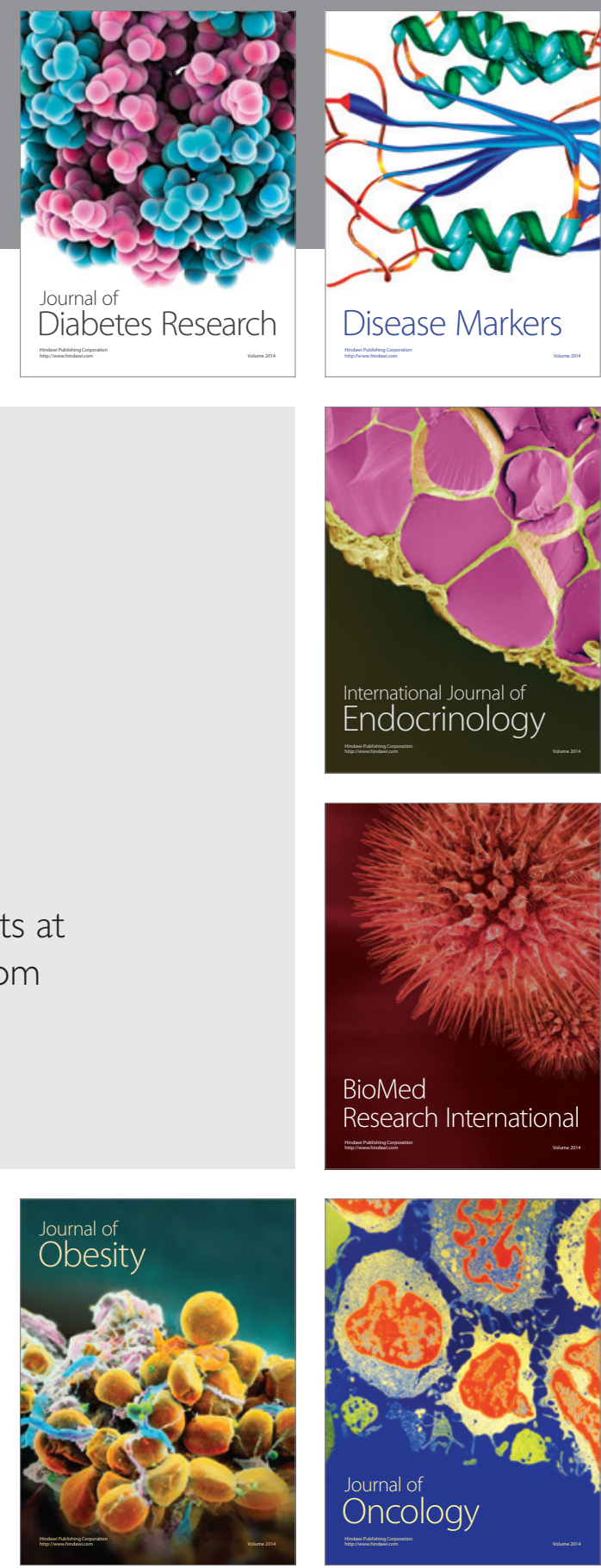

Disease Markers
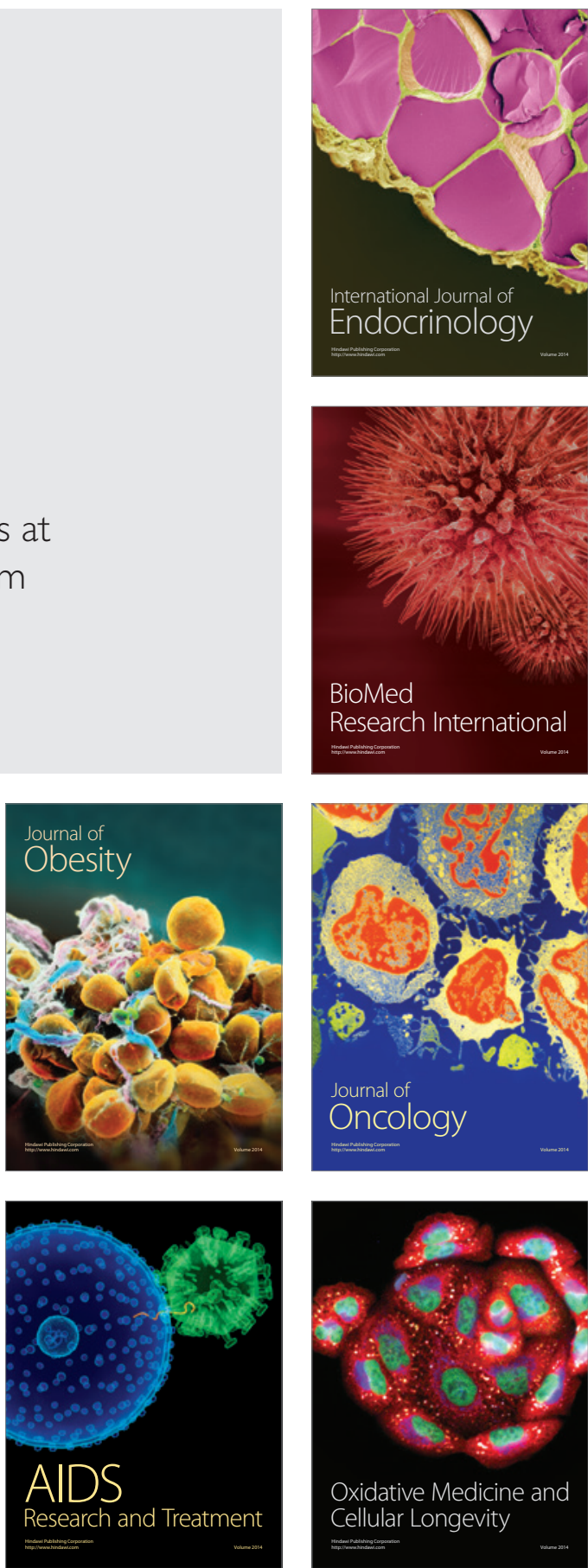